\title{
CORRECTION
}

\section{Correction to: Body composition assessment and sarcopenia in patients with gastric cancer: a systematic review and meta-analysis}

\author{
Sivesh K. Kamarajah ${ }^{1}\left[\right.$. James Bundred ${ }^{1} \cdot$ Benjamin H. L. $\operatorname{Tan}^{1}$
}

Published online: 1 March 2019

๑) The International Gastric Cancer Association and The Japanese Gastric Cancer Association 2019

\section{Correction to: Gastric Cancer https://doi.org/10.1007/s10120-018-0882-2}

In the original publication of this article, most of the reference citations in Tables 1 and 2 were published incorrectly. The corrected tables are given in this correction.

Table 1 Details of body composition assessment methodology

\begin{tabular}{|c|c|c|c|c|c|c|c|c|c|}
\hline Authors & Year & Region & Method & $\begin{array}{l}\text { Time of } \\
\text { assessment }\end{array}$ & $\begin{array}{l}\text { CT level of } \\
\text { assessment }\end{array}$ & $\begin{array}{l}\text { CT } \\
\text { muscle }\end{array}$ & $\begin{array}{l}\mathrm{CT} \\
\text { adipose } \\
\text { tissue }\end{array}$ & $\begin{array}{l}\text { Normali- } \\
\text { sation of } \\
\text { measure- } \\
\text { ments }\end{array}$ & Definition \\
\hline Kiyama [27] & 2005 & Asia & BIA & PreOp, PO & - & - & - & No & - \\
\hline Aoyama [14] & 2015 & Asia & BIA & PreOp, PO & - & - & - & No & - \\
\hline Fukuda [18] & 2015 & Asia & BIA & PreOp & - & - & - & No & $\begin{array}{l}8.87 \mathrm{~kg} / \mathrm{m}^{2} \text { (men), } \\
6.42 \mathrm{~kg} / \mathrm{m}^{2} \text { (women) }\end{array}$ \\
\hline Kobayashi [28] & 2015 & Asia & BIA & PreOp, PO & - & - & - & No & - \\
\hline Aoyama [15] & 2016 & Asia & BIA & PreOp, PO & - & - & - & No & - \\
\hline Sato [39] & 2016 & Asia & BIA & PreOp & - & - & - & No & $\begin{array}{l}7.25 \mathrm{~kg} / \mathrm{m}^{2} \text { (men), } \\
5.67 \mathrm{~kg} / \mathrm{m}^{2} \text { (women) }\end{array}$ \\
\hline $\begin{array}{l}\text { Yamamoto } \\
\text { [41] }\end{array}$ & 2017 & Asia & BIA & $\begin{array}{l}\text { Pre-Tx, } \\
\text { Post-Tx }\end{array}$ & - & - & - & Yes $(\mathrm{H})$ & $\begin{array}{l}43 \mathrm{~cm}^{2} / \mathrm{m}^{2}(\text { men with } \\
\left.\mathrm{BMI}<25.0 \mathrm{~kg} / \mathrm{m}^{2}\right), \\
53 \mathrm{~cm}^{2} / \mathrm{m}^{2}(\text { men with } \\
\left.\mathrm{BMI}>25 \mathrm{~kg} / \mathrm{m}^{2}\right), \\
41 \mathrm{~cm}^{2} / \mathrm{m}^{2} \text { (women) }\end{array}$ \\
\hline Aoyama [16] & 2018 & Asia & BIA & PreOp, PO & - & - & - & No & - \\
\hline Correia [47] & 2007 & Europe & BIA & PreOp & - & - & - & No & - \\
\hline Hiki [20] & 2009 & Asia & $\mathrm{CT}$ & PreOp & - & - & - & No & - \\
\hline Jeong [24] & 2009 & Asia & CT & PreOp & - & - & - & No & - \\
\hline Kunisaki [30] & 2011 & Asia & $\mathrm{CT}$ & PreOp & - & - & - & No & - \\
\hline Miyaki [35] & 2013 & Asia & CT & PreOp & - & - & - & No & - \\
\hline
\end{tabular}

The original article can be found online at https://doi.org/10.1007/ s10120-018-0882-2.

Benjamin H. L. Tan

benjamin.tan@uhb.nhs.uk

1 Department of Upper Gastrointestinal Surgery, Queen Elizabeth Hospital Birmingham, Birmingham, UK 


\begin{tabular}{|c|c|c|c|c|c|c|c|c|c|}
\hline Authors & Year & Region & Method & $\begin{array}{l}\text { Time of } \\
\text { assessment }\end{array}$ & $\begin{array}{l}\text { CT level of } \\
\text { assessment }\end{array}$ & $\begin{array}{l}\mathrm{CT} \\
\text { muscle }\end{array}$ & $\begin{array}{l}\mathrm{CT} \\
\text { adipose } \\
\text { tissue }\end{array}$ & $\begin{array}{l}\text { Normali- } \\
\text { sation of } \\
\text { measure- } \\
\text { ments }\end{array}$ & Definition \\
\hline Kim [26] & 2014 & Asia & $\mathrm{CT}$ & PreOp & $\mathrm{T} 10$ & - & $\begin{array}{l}(-) 190 \text { to } \\
-30\end{array}$ & No & - \\
\hline Yamaoka [42] & 2015 & Asia & $\mathrm{CT}$ & PreOp, PO & $\begin{array}{l}\text { L3 mid- } \\
\text { point }\end{array}$ & $\begin{array}{l}(-) 30 \text { to } \\
110\end{array}$ & $\begin{array}{l}(-) 190 \text { to } \\
-30\end{array}$ & No & - \\
\hline Chen [17] & 2016 & Asia & $\mathrm{CT}$ & PreOp & $\begin{array}{l}\text { L3 mid- } \\
\text { point }\end{array}$ & $\begin{array}{l}(-) 29 \text { to } \\
150\end{array}$ & - & Yes $(\mathrm{H})$ & $\begin{array}{l}<40.8 \mathrm{~cm}^{2} / \mathrm{m}^{2} \text { (men), } \\
\quad<34.9 \mathrm{~cm}^{2} / \mathrm{m}^{2} \\
\text { (women) }\end{array}$ \\
\hline Hayashi [19] & 2016 & Asia & $\mathrm{CT}$ & PreTx & $\begin{array}{l}\text { L3 mid- } \\
\text { point }\end{array}$ & $\begin{array}{c}(-) 29 \text { to } \\
150\end{array}$ & - & Yes $(H)$ & $\begin{array}{l}<43 \mathrm{~cm}^{2} / \mathrm{m}^{2} \text { (men), } \\
<41 \mathrm{~cm}^{2} / \mathrm{m}^{2} \text { (women) }\end{array}$ \\
\hline Huang [21] & 2016 & Asia & $\mathrm{CT}$ & PreOp & $\begin{array}{l}\text { L3 mid- } \\
\text { point }\end{array}$ & $\begin{array}{c}(-) 29 \text { to } \\
150\end{array}$ & - & Yes $(\mathrm{H})$ & $\begin{array}{l}<40.8 \mathrm{~cm}^{2} / \mathrm{m}^{2} \text { (men), } \\
<34.9 \mathrm{~cm}^{2} / \mathrm{m}^{2} \\
\quad \text { (women) }\end{array}$ \\
\hline Nishigori [37] & 2016 & Asia & $\mathrm{CT}$ & PreOp & $\begin{array}{l}\text { L3 mid- } \\
\text { point }\end{array}$ & $\begin{array}{l}(-) 29 \text { to } \\
150\end{array}$ & $\begin{array}{l}(-) 190 \text { to } \\
-30\end{array}$ & Yes $(\mathrm{H})$ & $\begin{aligned} \leq & 52.4 \mathrm{~cm}^{2} / \mathrm{m}^{2} \text { (men) } \\
& \leq 38.5 \mathrm{~cm}^{2} / \mathrm{m}^{2} \\
& \text { (women) }\end{aligned}$ \\
\hline Wang [40] & 2016 & Asia & $\mathrm{CT}$ & PreOp & $\begin{array}{l}\text { L3 mid- } \\
\text { point }\end{array}$ & $\begin{array}{l}(-) 29 \text { to } \\
150\end{array}$ & - & Yes $(\mathrm{H})$ & $\begin{array}{l}<36.0 \mathrm{~cm}^{2} / \mathrm{m}^{2} \text { (men) } \\
<29.0 \mathrm{~cm}^{2} / \mathrm{m}^{2} \\
\text { (women) }\end{array}$ \\
\hline Zhuang [45] & 2016 & Asia & $\mathrm{CT}$ & PreOp & $\begin{array}{l}\text { L3 mid- } \\
\text { point }\end{array}$ & $\begin{array}{l}(-) 29 \text { to } \\
150\end{array}$ & - & Yes $(\mathrm{H})$ & $\begin{array}{l}<40.8 \mathrm{~cm}^{2} / \mathrm{m}^{2} \text { (men) } \\
\quad<34.9 \mathrm{~cm}^{2} / \mathrm{m}^{2} \\
\quad \text { (women) }\end{array}$ \\
\hline Huang [23] & 2017 & Asia & $\mathrm{CT}$ & PreOp & $\begin{array}{l}\text { L3 mid- } \\
\text { point }\end{array}$ & $\begin{array}{l}\text { (-) } 29 \text { to } \\
150\end{array}$ & $\begin{array}{l}\text { (-) } 150 \text { to } \\
-50\end{array}$ & Yes $(\mathrm{H})$ & $\begin{array}{l}<40.8 \mathrm{~cm}^{2} / \mathrm{m}^{2} \text { (men) } \\
\quad<34.9 \mathrm{~cm}^{2} / \mathrm{m}^{2} \\
\text { (women) }\end{array}$ \\
\hline Huang [22] & 2017 & Asia & $\mathrm{CT}$ & PreOp, PO & $\begin{array}{l}\text { L3 mid- } \\
\text { point }\end{array}$ & $\begin{array}{l}(-) 29 \text { to } \\
150\end{array}$ & - & Yes $(\mathrm{H})$ & - \\
\hline Kudou [29] & 2017 & Asia & $\mathrm{CT}$ & PreOp & $\begin{array}{l}\text { L3 mid- } \\
\text { point }\end{array}$ & $\begin{array}{c}(-) 29 \text { to } \\
150\end{array}$ & - & Yes $(\mathrm{H})$ & $\begin{array}{l}43 \mathrm{~cm}^{2} / \mathrm{m}^{2}(\text { men with } \\
\left.\mathrm{BMI}<25.0 \mathrm{~kg} / \mathrm{m}^{2}\right), \\
53 \mathrm{~cm}^{2} / \mathrm{m}^{2}(\text { men with } \\
\left.\mathrm{BMI}>25 \mathrm{~kg} / \mathrm{m}^{2}\right), \\
41 \mathrm{~cm}^{2} / \mathrm{m}^{2} \text { (women) }\end{array}$ \\
\hline Lou [33] & 2017 & Asia & $\mathrm{CT}$ & PreOp & $\begin{array}{l}\text { L3 mid- } \\
\text { point }\end{array}$ & $\begin{array}{l}(-) 29 \text { to } \\
150\end{array}$ & - & Yes $(\mathrm{H})$ & $\begin{array}{l}<40.8 \mathrm{~cm}^{2} / \mathrm{m}^{2} \text { (men) } \\
\quad<34.9 \mathrm{~cm}^{2} / \mathrm{m}^{2} \\
\quad \text { (women) }\end{array}$ \\
\hline Nagata [36] & 2017 & Asia & $\mathrm{CT}$ & PreOp & $\begin{array}{l}\text { L3 mid- } \\
\text { point }\end{array}$ & - & - & Yes $(\mathrm{H})$ & - \\
\hline Sakurai [38] & 2017 & Asia & $\mathrm{CT}$ & PreOp & $\begin{array}{l}\text { L3 mid- } \\
\text { point }\end{array}$ & $\begin{array}{l}\text { (-) } 29 \text { to } \\
150\end{array}$ & - & Yes $(\mathrm{H})$ & $\begin{array}{l}<43.2 \mathrm{~cm}^{2} / \mathrm{m}^{2} \text { (men), } \\
\quad<34.6 \mathrm{~cm}^{2} / \mathrm{m}^{2} \\
\text { (women) }\end{array}$ \\
\hline Zheng [43] & 2017 & Asia & $\mathrm{CT}$ & PreOp & $\begin{array}{l}\text { L3 mid- } \\
\text { point }\end{array}$ & $\begin{array}{l}(-) 29 \text { to } \\
150\end{array}$ & - & Yes $(\mathrm{H})$ & $\begin{array}{l}<32.5 \mathrm{~cm}^{2} / \mathrm{m}^{2} \text { (men) } \\
\quad<28.6 \mathrm{~cm}^{2} / \mathrm{m}^{2} \\
\text { (women) }\end{array}$ \\
\hline Zhou [44] & 2017 & Asia & $\mathrm{CT}$ & PreOp & $\begin{array}{l}\text { L3 mid- } \\
\text { point }\end{array}$ & $\begin{array}{l}(-) 29 \text { to } \\
150\end{array}$ & - & Yes $(\mathrm{H})$ & $\begin{array}{l}<40.8 \mathrm{~cm}^{2} / \mathrm{m}^{2} \text { (men) } \\
\quad<34.9 \mathrm{~cm}^{2} / \mathrm{m}^{2} \\
\text { (women) }\end{array}$ \\
\hline Kugimiya [46] & 2018 & Asia & $\mathrm{CT}$ & PreOp, PO & $\begin{array}{l}\text { L3 mid- } \\
\text { point }\end{array}$ & $\begin{array}{l}(-) 30 \text { to } \\
110\end{array}$ & - & Yes $(\mathrm{H})$ & - \\
\hline Kuwada [31] & 2018 & Asia & $\mathrm{CT}$ & PreOp & $\begin{array}{l}\text { L3 mid- } \\
\text { point }\end{array}$ & $\begin{array}{c}(-) 30 \text { to } \\
150\end{array}$ & - & Yes (BSA) & $\begin{array}{l}69.7 \mathrm{~cm}^{2} / \mathrm{m}^{2} \text { (men), } \\
54.2 \mathrm{~cm}^{2} / \mathrm{m}^{2} \text { (women) }\end{array}$ \\
\hline $\mathrm{Lu}[34]$ & 2018 & Asia & $\mathrm{CT}$ & PreOp & $\begin{array}{l}\text { L3 mid- } \\
\text { point }\end{array}$ & $\begin{array}{c}(-) 30 \text { to } \\
110\end{array}$ & - & Yes $(\mathrm{H})$ & $\begin{array}{l}512.7 \mathrm{~mm}^{2} / \mathrm{m}^{2} \text { (men), } \\
344.3 \mathrm{~mm}^{2} / \mathrm{m}^{2} \\
\text { (women) }\end{array}$ \\
\hline
\end{tabular}




\begin{tabular}{|c|c|c|c|c|c|c|c|c|c|}
\hline Authors & Year & Region & Method & $\begin{array}{l}\text { Time of } \\
\text { assessment }\end{array}$ & $\begin{array}{l}\text { CT level of } \\
\text { assessment }\end{array}$ & $\begin{array}{l}\mathrm{CT} \\
\text { muscle }\end{array}$ & $\begin{array}{l}\mathrm{CT} \\
\text { adipose } \\
\text { tissue }\end{array}$ & $\begin{array}{l}\text { Normali- } \\
\text { sation of } \\
\text { measure- } \\
\text { ments }\end{array}$ & Definition \\
\hline Tegels [49] & 2015 & Europe & CT & PreOp & $\begin{array}{l}\text { L3 mid- } \\
\text { point }\end{array}$ & $\begin{array}{l}(-) 30 \text { to } \\
110\end{array}$ & $\begin{array}{l}(-) 190 \text { to } \\
-30\end{array}$ & Yes $(\mathrm{H})$ & $\begin{array}{l}43 \mathrm{~cm}^{2} / \mathrm{m}^{2} \text { (men with } \\
\left.\mathrm{BMI}<25.0 \mathrm{~kg} / \mathrm{m}^{2}\right), \\
53 \mathrm{~cm}^{2} / \mathrm{m}^{2}(\text { men with } \\
\left.\mathrm{BMI}>25 \mathrm{~kg} / \mathrm{m}^{2}\right), \\
41 \mathrm{~cm}^{2} / \mathrm{m}^{2} \text { (women) }\end{array}$ \\
\hline Palmela [5] & 2017 & Europe & CT & PreOp & $\begin{array}{l}\text { L3 mid- } \\
\text { point }\end{array}$ & $\begin{array}{l}(-) 29 \text { to } \\
150\end{array}$ & - & Yes $(\mathrm{H})$ & $\begin{array}{l}43 \mathrm{~cm}^{2} / \mathrm{m}^{2} \text { (men with } \\
\left.\mathrm{BMI}<25.0 \mathrm{~kg} / \mathrm{m}^{2}\right), \\
53 \mathrm{~cm}^{2} / \mathrm{m}^{2}(\text { men with } \\
\left.\mathrm{BMI}>25 \mathrm{~kg} / \mathrm{m}^{2}\right), \\
41 \mathrm{~cm}^{2} / \mathrm{m}^{2} \text { (women) }\end{array}$ \\
\hline Mirkin [51] & 2017 & $\begin{array}{l}\text { North } \\
\text { America }\end{array}$ & CT & $\begin{array}{l}\text { Pre-Tx, } \\
\text { Post-Tx }\end{array}$ & $\begin{array}{l}\text { L3 mid- } \\
\text { point }\end{array}$ & - & - & Yes $(\mathrm{H})$ & $\begin{array}{l}<545 \mathrm{~mm}^{2} / \mathrm{m}^{2} \text { (men) } \\
\quad<385 \mathrm{~mm}^{2} / \mathrm{m}^{2} \\
\quad \text { (women) }\end{array}$ \\
\hline Lee [32] & 2018 & Asia & DXA & PreOp, PO & - & - & - & No & - \\
\hline Liedman [48] & 1998 & Europe & DXA & PreOp, PO & - & - & - & No & - \\
\hline Copland [50] & 2007 & Europe & DXA & PreOp, PO & - & - & - & No & - \\
\hline $\begin{array}{l}\text { Kawamura } \\
\text { [25] }\end{array}$ & 2018 & Asia & TSF & PreOp & - & - & - & No & $\begin{array}{l}<38.05 \mathrm{~cm}^{2} \text { (men) } \\
<27.87 \mathrm{~cm}^{2} \text { (women) }\end{array}$ \\
\hline
\end{tabular}

CT muscle and adipose tissue reported as Hounsfield unit threshold

$B I A$ body impedance analysis, $B S A$ adjusted for body surface area, $C T$ computer tomography, $D X A$ dual-energy X-ray absorptiometry, $H$ adjusted for height, $P O$ post-operative, Post-Tx post-treatment (including neo-adjuvant therapy), PreOp pre-operative, $P r e-T x$ pre-treatment (including neo-adjuvant therapy), TSF thickness of skin fold

Table 2 Details of included studies

\begin{tabular}{|c|c|c|c|c|c|c|c|c|c|c|c|c|}
\hline Authors & Year & Method & Cancer site & Number & Design & $\begin{array}{l}\text { Age, } \\
\text { years }^{\mathrm{a}}\end{array}$ & $\begin{array}{l}\text { Sex }(\% \\
\text { male })\end{array}$ & $\begin{array}{l}\text { Extent of } \\
\text { resection }\end{array}$ & $\begin{array}{l}\text { T stage } \\
3 / 4, \%\end{array}$ & $\begin{array}{l}\text { Stage } \\
\text { III/IV, } \\
\%\end{array}$ & $\begin{array}{l}\text { Sarco- } \\
\text { penia } \\
\text { rates, \% }\end{array}$ & NOS \\
\hline Kiyama [27] & 2005 & BIA & - & 108 & RCS & - & 67 & $\begin{array}{c}\text { Total (45), } \\
\text { partial } \\
(63)\end{array}$ & - & 31 & - & 8 \\
\hline Aoyama [14] & 2015 & BIA & - & 58 & PCS & $67(36-80)^{\mathrm{b}}$ & 50 & $\begin{array}{c}\text { Total (31), } \\
\text { partial } \\
(27)\end{array}$ & - & 57 & - & 8 \\
\hline Fukuda [18] & 2015 & BIA & - & 99 & RCS & - & 67 & $\begin{array}{c}\text { Total (34), } \\
\text { partial } \\
(65)\end{array}$ & - & 30 & 21 & 9 \\
\hline $\begin{array}{l}\text { Kobayashi } \\
\text { [28] }\end{array}$ & 2017 & BIA & - & 82 & RCT & $67 \pm 9$ & 73 & $\begin{array}{c}\text { Total (25), } \\
\text { partial } \\
(57)\end{array}$ & - & 34 & - & $\mathrm{c}$ \\
\hline Aoyama [15] & 2016 & BIA & - & 485 & RCS & 68 & 62 & $\begin{array}{l}\text { Total } \\
\text { (190), } \\
\text { partial } \\
(295)\end{array}$ & 33 & - & - & 9 \\
\hline Sato [39] & 2016 & BIA & $\begin{array}{l}\text { Upper } \\
\text { (68), } \\
\text { middle } \\
(120), \\
\text { lower } \\
(107)\end{array}$ & 293 & RCS & 66 & 66 & - & - & 36 & 18 & 8 \\
\hline $\begin{array}{l}\text { Yamamoto } \\
\text { [41] }\end{array}$ & 2017 & BIA & - & 90 & PCS & $73 \pm 4$ & 58 & $\begin{array}{c}\text { Total (26), } \\
\text { partial } \\
(64)\end{array}$ & - & 21 & 24 & 6 \\
\hline
\end{tabular}




\begin{tabular}{|c|c|c|c|c|c|c|c|c|c|c|c|c|}
\hline Authors & Year & Method & Cancer site & Number & Design & $\begin{array}{l}\text { Age, } \\
\text { years }\end{array}$ & $\begin{array}{l}\text { Sex }(\% \\
\text { male) }\end{array}$ & $\begin{array}{l}\text { Extent of } \\
\text { resection }\end{array}$ & $\begin{array}{l}\text { T stage } \\
3 / 4, \%\end{array}$ & $\begin{array}{l}\text { Stage } \\
\text { III/IV, } \\
\%\end{array}$ & $\begin{array}{l}\text { Sarco- } \\
\text { penia } \\
\text { rates, \% }\end{array}$ & NOS \\
\hline Aoyama [16] & 2018 & BIA & $\begin{array}{l}\text { Upper } \\
\text { (46), } \\
\text { middle } \\
(57)\end{array}$ & 103 & $\mathrm{RCT}$ & $\begin{array}{l}65 \\
(36-78)^{b}\end{array}$ & 58 & $\begin{array}{r}\text { Partial } \\
(103)\end{array}$ & 0 & 0 & - & c \\
\hline Correia [47] & 2007 & BIA & $\begin{array}{l}\text { Upper } \\
(32), \\
\text { middle } \\
(14), \\
\text { lower (2) }\end{array}$ & 48 & PCS & - & - & - & - & 33 & - & 6 \\
\hline Hiki [20] & 2009 & $\mathrm{CT}$ & $\begin{array}{l}\text { Lower } \\
(154)\end{array}$ & 154 & RCS & $60 \pm 1$ & 54 & $\begin{array}{l}\text { Partial } \\
(154)\end{array}$ & - & 1 & - & 6 \\
\hline Jeong [24] & 2009 & $\mathrm{CT}$ & Lower (80) & 80 & RCS & 57 (NA) & 68 & $\begin{array}{l}\text { Partial } \\
\quad(80)\end{array}$ & - & - & - & 6 \\
\hline Kunisaki [30] & 2011 & $\mathrm{CT}$ & - & 45 & RCS & $66 \pm 10$ & 62 & Total (45) & - & - & - & 7 \\
\hline Miyaki [35] & 2013 & CT & Lower (84) & 84 & RCS & $67 \pm 11$ & 61 & $\begin{array}{l}\text { Partial } \\
\text { (84) }\end{array}$ & - & 7 & - & 8 \\
\hline Kim [26] & 2014 & $\mathrm{CT}$ & - & 304 & RCS & $\begin{array}{l}60 \\
(25-86)^{b}\end{array}$ & 68 & $\begin{array}{c}\text { Total (74), } \\
\text { partial } \\
(230)\end{array}$ & 41 & 24 & - & 8 \\
\hline $\begin{array}{c}\text { Yamaoka } \\
\text { [42] }\end{array}$ & 2015 & $\mathrm{CT}$ & - & 102 & RCS & $64 \pm 11$ & 70 & $\begin{array}{l}\text { Total } \\
\text { (102) }\end{array}$ & - & 24 & - & c \\
\hline Chen [17] & 2016 & $\mathrm{CT}$ & $\begin{array}{l}\text { Upper } \\
(54), \\
\text { others } \\
(104)\end{array}$ & 158 & PCS & $67 \pm 9$ & 80 & - & 78 & 56 & 25 & 8 \\
\hline Hayashi [19] & 2016 & $\mathrm{CT}$ & - & 53 & RCS & - & 62 & $\begin{array}{l}\text { Total } \\
\text { (34), no } \\
\text { surgery } \\
(19)\end{array}$ & 0 & - & 70 & 8 \\
\hline Huang [21] & 2016 & $\mathrm{CT}$ & $\begin{array}{l}\text { Upper } \\
\text { (24), } \\
\text { middle } \\
(40), \\
\text { lower } \\
(98), \\
\text { mixed } \\
(11)\end{array}$ & 173 & PCS & $72 \pm 8$ & 78 & $\begin{array}{l}\text { Total }(71) \\
\text { subtotal } \\
(10)\end{array}$ & - & 46 & 30 & 9 \\
\hline $\begin{array}{l}\text { Nishigori } \\
\text { [37] }\end{array}$ & 2016 & $\mathrm{CT}$ & - & 157 & RCS & - & 66 & $\begin{array}{l}\text { Total } \\
\text { (157) }\end{array}$ & 42 & 24 & 62 & 9 \\
\hline Wang [40] & 2016 & $\mathrm{CT}$ & - & 255 & RCS & $65 \pm 11$ & 75 & $\begin{array}{l}\text { Total }(85) \text {, } \\
\text { subtotal } \\
(170)\end{array}$ & - & 49 & 13 & 9 \\
\hline Zhuang [45] & 2016 & CT & $\begin{array}{l}\text { Upper } \\
\text { (178), } \\
\text { NOS } \\
(759)\end{array}$ & 937 & RCS & $64 \pm 15$ & 78 & $\begin{array}{l}\text { Total } \\
\text { (337), } \\
\text { subtotal } \\
(600)\end{array}$ & 65 & 48 & 42 & 9 \\
\hline Huang [23] & 2017 & $\mathrm{CT}$ & $\begin{array}{l}\text { Upper } \\
\text { (60), } \\
\text { middle } \\
(112), \\
\text { lower } \\
(281), \\
\text { mixed } \\
(17)\end{array}$ & 391 & RCS & $65 \pm 15$ & 93 & $\begin{array}{l}\text { Total } \\
\text { (164), } \\
\text { subtotal } \\
(306)\end{array}$ & - & 52 & 20 & 7 \\
\hline Huang [22] & 2017 & $\mathrm{CT}$ & - & 110 & PCS & $63 \pm 10$ & 74 & $\begin{array}{c}\text { Total (31), } \\
\text { partial } \\
\text { (79) }\end{array}$ & 38 & - & - & 9 \\
\hline
\end{tabular}




\begin{tabular}{|c|c|c|c|c|c|c|c|c|c|c|c|c|}
\hline Authors & Year & Method & Cancer site & Number & Design & $\begin{array}{l}\text { Age, } \\
\text { years }^{\mathrm{a}}\end{array}$ & $\begin{array}{l}\text { Sex }(\% \\
\text { male) }\end{array}$ & $\begin{array}{l}\text { Extent of } \\
\text { resection }\end{array}$ & $\begin{array}{l}\text { T stage } \\
3 / 4, \%\end{array}$ & $\begin{array}{l}\text { Stage } \\
\text { III/IV, } \\
\%\end{array}$ & $\begin{array}{l}\text { Sarco- } \\
\text { penia } \\
\text { rates, \% }\end{array}$ & NOS \\
\hline Kudou [29] & 2017 & CT & $\begin{array}{l}\text { Upper } \\
\text { (89), } \\
\text { mixed } \\
(59)\end{array}$ & 148 & RCS & $\begin{array}{l}68 \\
(52-87)^{b}\end{array}$ & 72 & $\begin{array}{l}\text { Total } \\
\text { (143), } \\
\text { subtotal } \\
(5)\end{array}$ & 48 & 26 & 28 & 9 \\
\hline Lou [33] & 2017 & CT & - & 206 & PCS & $64 \pm 10$ & 8 & $\begin{array}{c}\text { Total (65), } \\
\text { subtotal } \\
(141)\end{array}$ & - & 39 & 7 & 8 \\
\hline Nagata [36] & 2017 & CT & $\begin{array}{l}\text { Upper (8), } \\
\text { middle } \\
(37), \\
\text { lower } \\
(31)\end{array}$ & 77 & RCS & $69 \pm 2$ & 47 & $\begin{array}{l}\text { Total (15), } \\
\text { partial } \\
(62)\end{array}$ & - & - & - & 8 \\
\hline Sakurai [38] & 2017 & CT & - & 569 & RCS & $67 \pm 11$ & 70 & $\begin{array}{l}\text { Total } \\
\text { (203), } \\
\text { subtotal } \\
(366)\end{array}$ & 45 & 32 & 25 & 9 \\
\hline Zheng [43] & 2017 & CT & - & 693 & RCS & $61 \pm 11$ & 76 & - & - & - & 15 & 9 \\
\hline Zhou [44] & 2017 & CT & $\begin{array}{l}\text { Upper } \\
\text { (37), } \\
\text { middle } \\
(52), \\
\text { lower } \\
\text { (139), } \\
\text { mixed } \\
(12)\end{array}$ & 240 & PCS & $73 \pm 7$ & 79 & $\begin{array}{c}\text { Total (97), } \\
\text { subtotal } \\
(143)\end{array}$ & - & 46 & 29 & 9 \\
\hline $\begin{array}{c}\text { Kugimiya } \\
{[46]}\end{array}$ & 2018 & CT & - & 119 & RCS & $70 \pm 10$ & 67 & $\begin{array}{c}\text { Total (45), } \\
\text { partial } \\
(74)\end{array}$ & 36 & 21 & - & 9 \\
\hline Kuwada [31] & 2018 & CT & - & 491 & RCS & $68 \pm 10$ & 71 & - & - & 22 & 25 & 9 \\
\hline Lu [34] & 2018 & $\mathrm{CT}$ & $\begin{array}{l}\text { Upper } \\
(52), \\
\text { middle } \\
(33), \\
\text { lower } \\
(109), \\
\text { mixed } \\
(27)\end{array}$ & 221 & PCS & ${ }^{62}(31-89)^{\mathrm{b}}$ & 76 & $\begin{array}{l}\text { Total } \\
\text { (111), } \\
\text { partial } \\
(110)\end{array}$ & 44 & - & 25 & 9 \\
\hline Tegels [49] & 2015 & CT & - & 149 & RCS & $\begin{array}{l}70 \\
(37-88)^{\mathrm{b}}\end{array}$ & 58 & $\begin{array}{l}\text { Total (40), } \\
\text { subtotal } \\
(84), \\
\text { others } \\
(28)\end{array}$ & - & 58 & 58 & 9 \\
\hline Palmela [5] & 2017 & CT & $\begin{array}{l}\text { Upper (5), } \\
\text { middle } \\
(23), \\
\text { lower } \\
(19)\end{array}$ & 47 & RCS & $68 \pm 10$ & 68 & - & - & 89 & 23 & 8 \\
\hline Mirkin [51] & 2017 & $\mathrm{CT}$ & Body (18) & 36 & RCS & 65 (NA) & 36 & $\begin{array}{l}\text { Total (26), } \\
\text { subtotal } \\
(10)\end{array}$ & - & - & 33 & 8 \\
\hline Lee [32] & 2018 & DXA & - & 37 & PCS & $56 \pm 11$ & 65 & $\begin{array}{c}\text { Total (2), } \\
\text { partial } \\
(35)\end{array}$ & 0 & 0 & - & 6 \\
\hline Liedman [48] & 1998 & DXA & $\begin{array}{l}\text { Upper } \\
\text { (13), } \\
\text { lower } \\
(26)\end{array}$ & 36 & RCT & 63 (NA) & 67 & $\begin{array}{l}\text { Total (33), } \\
\text { partial } \\
(3)\end{array}$ & 17 & - & - & c \\
\hline Copland [50] & 2007 & DXA & - & 13 & PCS & $68 \pm 8$ & 69 & Total (13) & - & - & - & c \\
\hline
\end{tabular}




\begin{tabular}{lllllllllll}
\hline Authors & Year & Method & Cancer site Number Design & $\begin{array}{l}\text { Age, } \\
\text { years }\end{array}$ & $\begin{array}{l}\text { Sex (\% } \\
\text { male) }\end{array}$ & $\begin{array}{l}\text { Extent of } \\
\text { resection }\end{array}$ & $\begin{array}{l}\text { T stage } \\
3 / 4, \%\end{array} \% \begin{array}{l}\text { Stage } \\
\text { III/IV, } \\
\%\end{array}$ & $\begin{array}{l}\text { Sarco- } \\
\text { penia } \\
\text { rates, } \%\end{array}$ & $\begin{array}{l}\text { NOS } \\
\%\end{array}$ \\
\hline $\begin{array}{l}\text { Kawamura } \\
{[25]}\end{array}$ & 2018 & TSF & - & 951 & RCS & - & 69 & - & - & - \\
\hline
\end{tabular}

$B I A$ body impedance analysis, $B S A$ adjusted for body surface area, $C T$ computer tomography, $D X A$ dual-energy X-ray absorptiometry, $H$ adjusted for height, $N A$ not available, NOS Newcastle-Ottawa Score, $P C S$ prospective cohort study, $P O$ post-operative, Post-Tx post-treatment (including neo-adjuvant therapy), PreOp pre-operative, Pre-Tx pre-treatment (including neo-adjuvant therapy), $R C T$ randomised controlled trial, $R C S$ retrospective cohort study, TSF thickness of skin fold

${ }^{\text {a }}$ Data presented as mean \pm standard deviation unless otherwise stated

${ }^{\mathrm{b}}$ Median (range)

${ }^{\mathrm{c}} \mathrm{NOS}$ only applies to cohort

Publisher's Note Springer Nature remains neutral with regard to jurisdictional claims in published maps and institutional affiliations. 